\title{
BMJ Open Effectiveness of adult community-based physical activity interventions with objective physical activity measurements and long-term follow-up: a systematic review and meta-analysis
}

Charlotte Wahlich, ${ }^{1,2}$ Umar A R Chaudhry, ${ }^{1}$ Rebecca Fortescue, ${ }^{1}$ Derek G Cook, ${ }^{1}$ Shashivadan Hirani, ${ }^{2}$ Rachel Knightly, ${ }^{1}$ Tess Harris (D) ${ }^{1}$

To cite: Wahlich C,

Chaudhry UAR, Fortescue R, et al. Effectiveness of adult community-based physical activity interventions with objective physical activity measurements and long-term follow-up: a systematic review and meta-analysis. BMJ Open 2020;10:e034541. doi:10.1136/ bmjopen-2019-034541

- Prepublication history and additional material for this paper are available online. To view these files, please visit the journal online (http://dx.doi. org/10.1136/bmjopen-2019034541).

Received 24 September 2019 Revised 18 January 2020 Accepted 02 April 2020
Check for updates

(C) Author(s) (or their employer(s)) 2020. Re-use permitted under CC BY-NC. No commercial re-use. See rights and permissions. Published by BMJ.

${ }^{1}$ Population Health Research Institute, St George's, University of London, London, UK

${ }^{2}$ School of Health Sciences, City University, London, UK

Correspondence to

Dr Tess Harris;

tharris@sgul.ac.uk

\section{ABSTRACT}

Objective To identify randomised controlled trials (RCTS) of physical activity (PA) interventions with objective PA outcomes in adults and to evaluate whether intervention effects were sustained beyond 12 months.

Design Systematic review and meta-analysis.

Data sources Seven databases (Medline, Embase, PsycINF0, Web of Science, Cochrane library, CINAHL (Cumulative Index of Nursing and Allied Health Literature) and ASSIA (Applied Social Sciences Index and Abstracts)) were searched from January 2000 until December 2019. Eligibility criteria RCTs reporting objective PA outcomes beyond 12 months with community-based participants aged $\geq 18$ years were included; those where controls received active interventions, including advice to increase PA levels, were excluded.

Data extraction and synthesis Two independent reviewers completed extraction of aggregate data and assessed risk of bias. Meta-analyses used random-effects models at different follow-up points. Primary outcomes were daily steps and weekly minutes of moderate-tovigorous PA (MVPA).

Results 0 33282 records identified, nine studies (at generally low risk of bias) were included, five in meta-analyses with 12 months to 4 year follow-up. We observed 12 month increases for intervention vs control participants in steps/day (mean difference $(\mathrm{MD})=554$ (95\% Cls: 384 to 724$) \mathrm{p}<0.0001, \mathrm{l}^{2}=0 \%$; 2446 participants; four studies) and weekly MVPA minutes (MD=35 (95\% Cl: 27 to 43) $p<0.0001, l^{2}=0 \%$; 2647 participants; four studies). Effects were sustained up to 4 years for steps/day (MD=494 (95\% Cl: 251 to 738) $p<0.0001, I^{2}=0 \%$; 1944 participants; four studies) and weekly MVPA minutes (MD=25 (95\% Cl: 13 to 37) $\mathrm{p}<0.0001, \mathrm{I}^{2}=0 \%$; 1458 participants; three studies). Conclusions There are few PA interventions with objective follow-up beyond 12 months, more studies are needed. However, this review provided evidence of PA intervention effects beyond 12 months and sustained up to 4 years for both steps/day and MVPA. These findings have important implications for potential long-term health benefits.

PROSPERO registration number CRD42017075753.
Strengths and limitations of this study

- Prepublication of our protocol on PROSPERO ensures methodological transparency and mitigates against potential post-hoc decision making.

- Study selection, data extraction and quality assessments were conducted independently by two reviewers using standardised forms.

- We were able to perform meta-analysis to evaluate our primary aims.

- The generalisability of our evidence is restricted as few studies were identified and all were conducted in high-income countries, with the majority of participants of white ethnicity and aged over 40 years old.

- Small study numbers also meant we were unable to perform subgroup and sensitivity analyses to evaluate our secondary aims relating to which physical activity interventions/intervention components were most effective.

\section{INTRODUCTION}

Physical activity (PA) is associated with important health benefits such as reducing premature mortality and preventing and managing several chronic medical conditions. ${ }^{12}$ However, more than a quarter of people worldwide fail to meet the guidelines of $150 \mathrm{~min}$ of moderate-to-vigorous PA (MVPA) in $\geq 10$ min bouts weekly. ${ }^{34}$ Physical inactivity costs the UK economy $£ 4$. 7 billion $^{5}$ and the US healthcare system US $\$ 117$ billion annually. ${ }^{4}$

Systematic review evidence indicates that different PA interventions, including pedometer-based ${ }^{6}$ and individual and groupbased interventions, ${ }^{7}$ increase PA levels in the short term. However, for health benefits, PA needs to be maintained. ${ }^{8}$ Currently little is known about the long-term sustainability of PA interventions. A meta-analysis, ${ }^{9}$ 
which examined the long-term effects of behavioural PA interventions, included only two trials with objective data beyond 12 months ${ }^{10} 11$ and more trials with longer-term follow-up and objective PA measurements are needed. ${ }^{912}$

Accurate PA assessment is needed to determine the relationship between $\mathrm{PA}$ and health and avoid bias and misclassification. ${ }^{13}$ Subjective self-report PA questionnaires are susceptible to inaccuracy through social desirability, ${ }^{14}$ recall bias ${ }^{15}$ or cognitive impairment. ${ }^{16}$ Directly compared self-reported and objective PA levels have shown considerable discrepancy, with self-reported over-estimating PA. ${ }^{17} 18$ Also, when measuring change in PA levels, accelerometry minimises bias and improves precision compared with self-report. ${ }^{1918}$ Pedometers are popular, simple and low-cost objective measurement devices. Accelerometers can capture both step-counts and time spent in different PA intensities, while remaining blind to participants. ${ }^{20}$ More trials now measure PA objectively; the proportion of studies using objective PA measures has grown from approximately $4 \%$ in 2006 to $71 \%$ in $2016 . .^{20}$

The primary aims were to identify and describe trials in adults with objective PA measures and long-term follow-up ( $\geq 12$ months) and to determine whether the PA intervention effects varied with follow-up beyond 12 months. Pending sufficient data, secondary aims were to:

- Determine which interventions are more effective at improving objectively measured PA outcomes beyond 12 months.

- Evaluate whether, and to what extent, different trial PA components affected adults' overall PA.

- Identify potential mediators/moderators of PA maintenance.

\section{METHODS}

The protocol for this systematic review was registered with the International Prospective Register of Systematic Reviews. The review follows the Preferred Reporting Items for Systematic Reviews and Meta-Analysis Guidelines.

\section{Search strategy}

We searched seven databases (Medline, Embase, PsycINFO, Web of Science, Cochrane library, CINAHL and ASSIA) using a combination of three key blocks of terms (PA, objective PA measures and randomised controlled trial, RCT) involving Medical Subject Heading terms and text words (online supplementary additional file 1 and 2). Initial search performed September 2017, with updated searches performed April 2018 and December 2019

We checked reference lists of all primary studies and reviews for additional eligible papers.

Study selection, summary estimates data extraction and risk-of-bias assessments were done independently by two reviewers. Conflicts were resolved via discussion until agreement reached; if necessary a third reviewer was consulted.

\section{Inclusion and exclusion criteria}

We included RCTs published in English after 01 January 2000. Participants were aged $\geq 18$ years old, healthy, or those 'at risk' of chronic diseases or those with preexisting chronic medical conditions (either physical or psychological) as expected within a general population. We excluded studies focusing on specific health conditions for example, diabetes, heart disease, etc. We included trials with community-based interventions that objectively measured PA (eg, steps/day, weekly minutes of MVPA) as outcomes, with follow-up beyond 12 months. We excluded trials where control groups received active interventions, including advice about increasing their PA levels (online supplementary additional file 3 ).

\section{Data extraction}

We imported search results into EndNote V.X7.7.1. After de-duplication, we applied an RCT classifier, ${ }^{21}$ excluding those with $0 \%-5 \%$ likelihood of being an RCT. Remaining titles and abstracts were screened independently by two of four reviewers (RF/CW and UARC/TH) using a checklist (online supplementary additional file 4). Full-texts of potentially relevant studies were assessed independently by the same reviewers. Discrepancies were resolved by discussions, if necessary consulting a third reviewer (TH or DGC as appropriate).

The following data from the nine included studies were extracted independently by RF/CW using a pre-piloted data extraction form: demographic details (age, sex, ethnicity and health status); trial duration and setting; intervention and comparator details; method of outcome measurement; and outcome data.

We contacted three investigators to verify key study characteristics and obtain missing numerical outcome data, obtaining additional unpublished data from two. ${ }^{22} 23$ Newman $e t a l^{24}$ were unable to provide estimated treatment effects at different time points. We did not contact Suguira et $a l^{25}$ authors as it was a small study $(\mathrm{n}=48)$ conducted 17 years ago. These two latter studies were included in the narrative review.

\section{Quality assessment}

Two reviewers (from CW/UARC/RK) assessed risk of bias independently for each study using the seven domains outlined in the Cochrane Handbook. ${ }^{26} \mathrm{CW}$ was research assistant on PACE-UP, therefore PACE-UP and PACE-Lift trials $^{27}$ were independently assessed by RK/UARC. Any disagreements were resolved through discussion with a fourth reviewer (RF).

We judged each domain as either presenting a high, low or unclear risk of bias. A summary is provided (online supplementary additional file 5) with supporting quotes and justifications (online supplementary additional file 6).

\section{Statistical analysis}

We analysed continuous data using change in mean differences (MDs) from baseline in our meta-analyses using Review Manager V. 5.3. We extracted outcomes from 


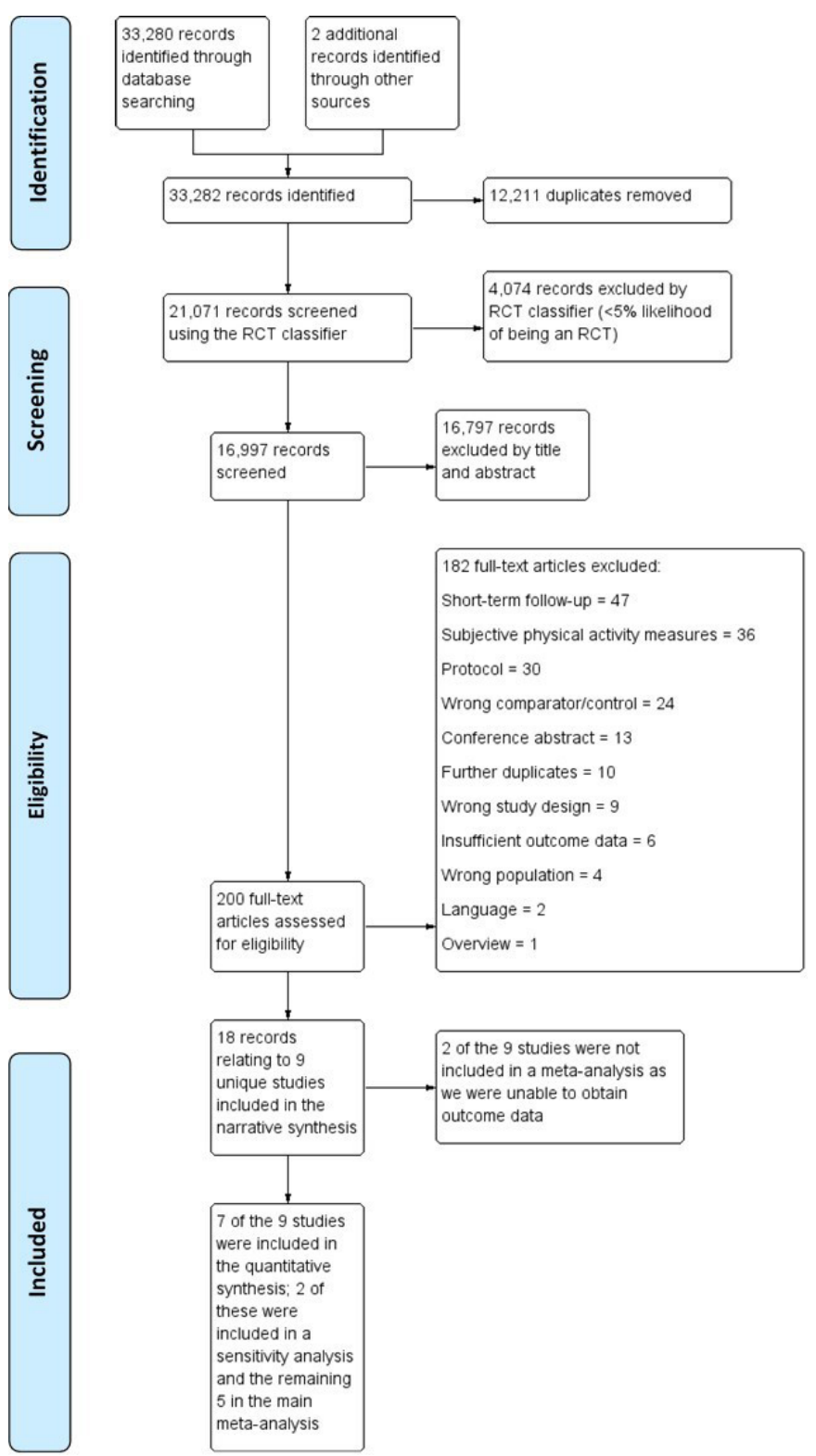

Figure 1 PRISMA flowchart of literature search results.

all available time points (including prior to 12 months) to examine trends over follow-up. As we anticipated between-study heterogeneity, a random-effects model was used. For trials with multiple relevant arms, we performed necessary adjustments to the data first (eg, splitting the comparator group to avoid double-counting for Harris et $a l^{27}$ PACE-UP). Meta-analyses were conducted in Review Manager V. 5.3.

We used the $\mathrm{I}^{2}$ statistic to measure statistical heterogeneity among the studies in each analysis; we used the cut offs $<30 \%, 30 \%-60 \%, 61 \%-75 \%$ and $>75 \%$ suggesting low, moderate, substantial and considerable heterogeneity, respectively. ${ }^{28}$

No intervention effects were seen at 12 months for both Varma 2016 (step-count) and Hays et $a l^{23}$ (MVPA). As this review focused on PA maintenance beyond 12 months, these studies were excluded from our final meta-analysis, but included in a sensitivity analysis. We present sensitivity analysis of the total MD in daily steps and weekly MVPA, at the different time points, including and excluding these studies (online supplementary additional file 7 ).

As steps/day and weekly minutes of MVPA are strongly correlated within studies, and outcomes at different time points are also moderately correlated within study, we carried out a single multivariate random-effects metaanalysis using Stata routine mvmeta ${ }^{29} 30$ which pooled data from all studies at all time-points for both steps/day and MVPA. The intention was to provide a clearer and more precise overview, taking account of all the data for both outcomes and all time-points. The methods used are described in online supplementary additional file 8 .

\section{Patient and public involvement}

Patients and the public were not involved in this review.

\section{RESULTS}

\section{Search results}

We identified 33282 records; after de-duplications and using the RCT classifier 16997 were screened. Two hundred full-text articles were assessed for eligibility, of these 18 records relating to nine unique studies met the inclusion criteria. Five of the nine studies were included in the main meta-analyses and two in sensitivity analyses. Figure 1 presents the study selection process flow-chart including reasons for exclusions.

\section{Study characteristics}

Details of eligible studies are presented in table 1 . The nine included RCTs randomly assigned 5832 participants to comparisons of interest. The largest study included 1635 participants, the smallest 48; mean number of participants 648, median number 509.

Four studies were conducted in the UK, four in the USA and one in Japan. Most recruited participants through primary care, two via the community, one did not report their recruitment method. Length of follow-up varied; one study was 18 months, four studies 2 years, three studies 3 years and one study 4 years. Five studies measured PA using ActiGraph accelerometers; one using a Step Activity Monitor accelerometer; and three using pedometers.

Most studies recruited adults aged $\geq 40$ years, three focused on adults aged $\geq 60$ years, three recruited participants at risk of type 2 diabetes mellitus. Three studies recruited participants with low baseline PA: Harris et $a l^{27}$ PACE-UP recruited participants self-reporting engaging in $<150$ min of MVPA weekly; Suguira et $a l^{25}$ recruited participants not engaging in regular exercise; and Pahor et $a l^{22}$ recruited 'inactive' participants. Most participants were of white ethnicity. Control groups did not receive specific instructions about increasing their PA levels, but in three studies they received a booklet or handout on type 2 diabetes mellitus risk reduction and in two studies they received educational courses or workshops on successful ageing. 


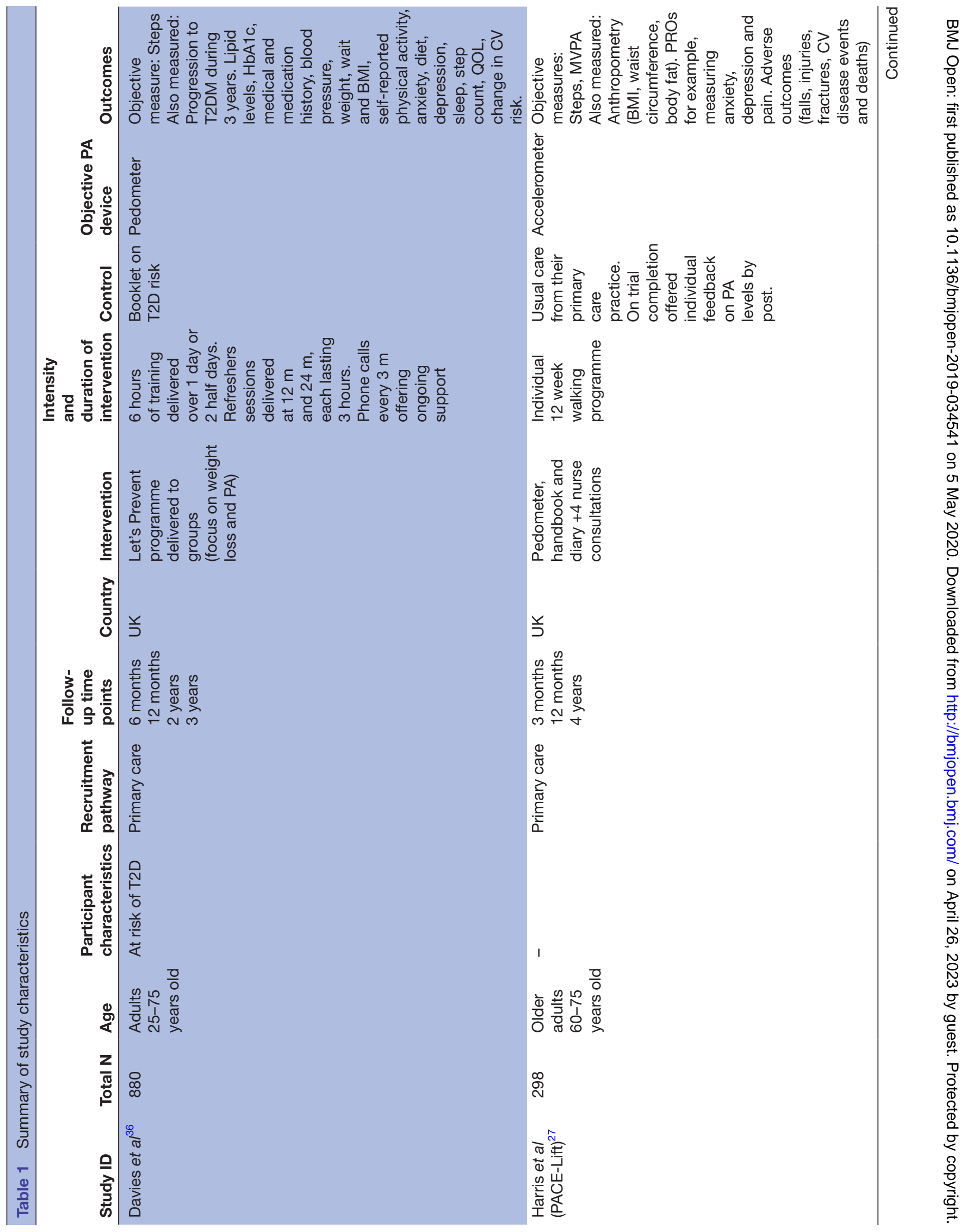




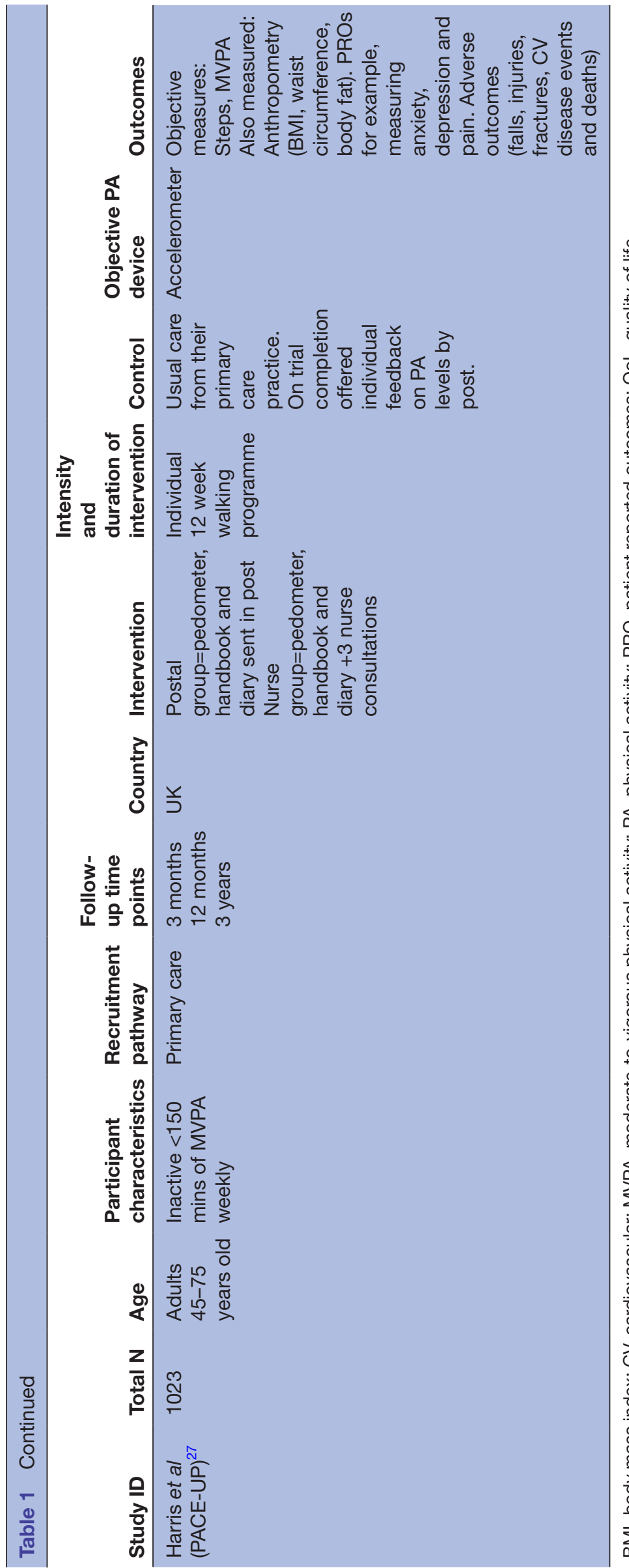

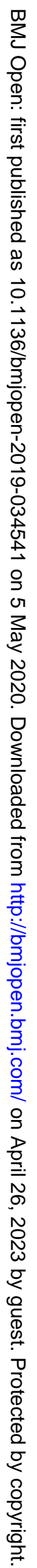




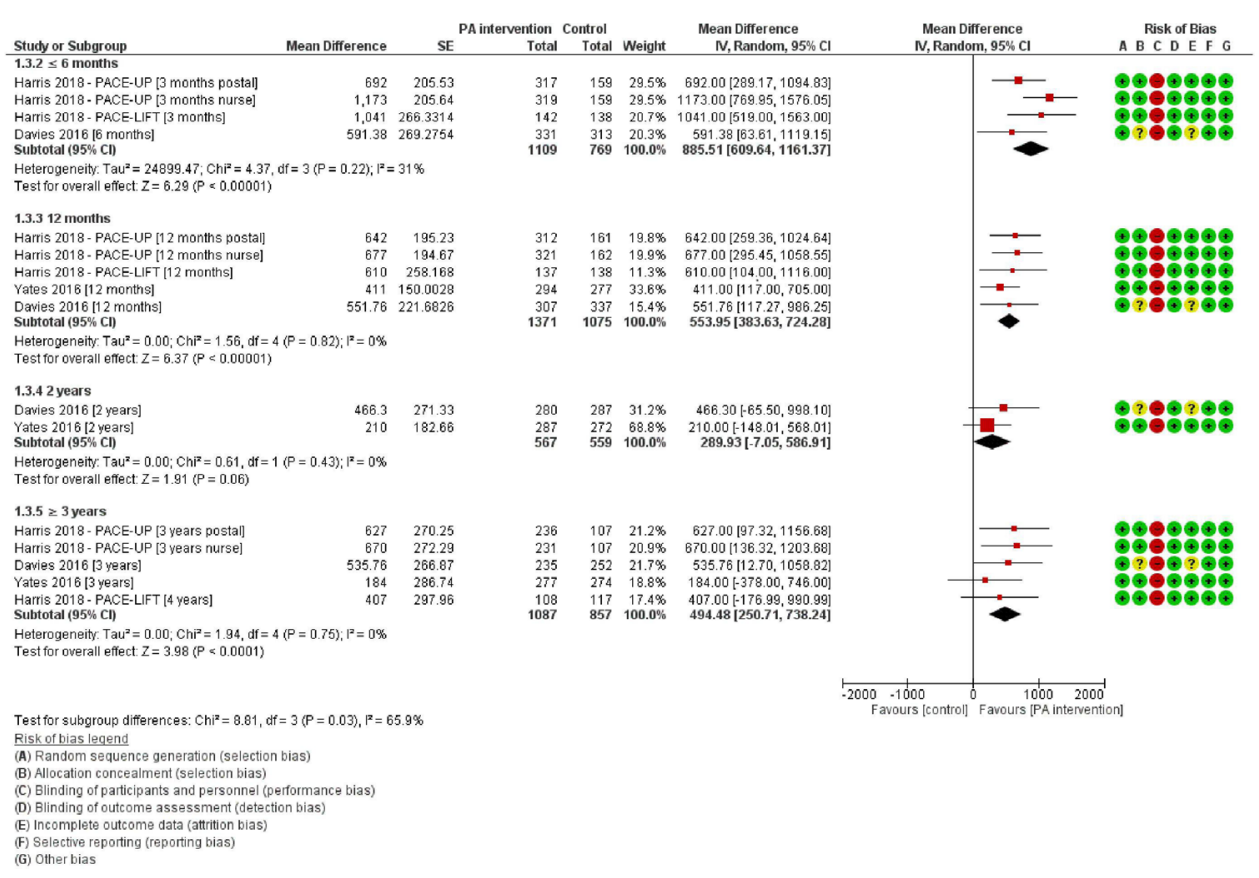

Figure 2 Forest plot showing mean change in daily steps.

All included studies aimed to increase PA, particularly walking. Harris $e t a l^{27}$ PACE-UP and Harris et $a l^{27}$ PACELift delivered individual level pedometer based-walking programmes and Pahor et $a l^{22}$ delivered individualised PA sessions at a centre, as well as home-based activities, whereas five studies delivered group interventions. Varma et $a \hat{l}^{\beta}$ was a little different in evaluating the effect of volunteering in schools on older adults' walking. Three interventions also included dietary advice.

Intervention length and intensity varied considerably; some were delivered weekly/ fortnightly over a year, ${ }^{22}$ whereas others had shorter time-frames, for example, 12 weeks in Harris $e t a l^{27}$ PACE-UP/Harris $e t a l^{27}$ PACE-Lift. Full intervention details are provided (table 1).

Three studies reported step-count and MVPA, four studies step-count only and two MVPA only. Studies reporting MVPA used different counts/minute cut-off points; Harris $e t a l^{27}$ PACE-UP, Harris $e t a l^{27}$ PACE-Lift and Yates $e t a l^{2}$ all defined MVPA as $\geq 1952$ counts/minute whereas Pahor et $a l^{22}$ and Hays $e t a l^{23}$ defined 'moderate $\mathrm{PA}^{\prime}$ as $\geq 760$ counts $/ \mathrm{min}$.

Most studies were funded by governmental agencies for example, National Institute for Health Research, National Heart, Lung and Blood Institute.

\section{Risk of bias of included studies}

Risk of bias judgements are presented in figures 2 and 3 . All but one study adequately described the generation of a randomization sequence and were therefore judged to be at low risk of bias in this domain. Suguira $e t a l^{25}$ was judged to be at high risk, as it was unclear how groups were 'randomly divided'. Allocation concealment was considered low risk for six studies, but unclear for three, due to insufficient detail. All studies were judged to be at high risk of performance bias, as the interventions made blinding unlikely. Hays $e t a t^{23}$ were judged to have a low risk of bias for participant performance bias, but a high risk for personnel, therefore, we assigned a high overall risk of performance bias. Given the objective outcome measures, seven studies were judged to be at low risk of detection bias, as they measured PA using an accelerometer or sealed pedometer. One study was considered high risk because participants recorded their own step-counts. ${ }^{24}$ Suguira et $a l^{25}$ provided insufficient details and was judged to have an unclear risk. Studies varied in the level of risk of attrition bias. We judged Harris et $a l^{27}$ PACE-Lift, Harris et $a l^{27}$ PACE-UP, Varma 2016 and Yates $e t a l^{32}$ to be at low risk: attrition was balanced for each trial arm, reasons for drop-outs were provided and intention-to-treat sensitivity analyses were performed. Suguira $e t a l^{25}$ was judged to be at high risk of attrition bias due to differential completion between trial arms and not conducting intention-to-treat analysis. The remaining four studies provided insufficient information and had an unclear risk of attrition bias. For eight studies the level of risk of reporting bias was low, for one study (Suguira $e t a l^{25}$ ) the risk was high, as it lacked prospective registration or a published protocol.

\section{Effects of interventions}

Five studies were included in the final meta-analyses. Harris $e t a l^{27}$ PACE-UP had two intervention arms (nurse and postal), which were presented separately in the meta-analyses.

\section{Difference in mean change in steps/day}

Figure 2 shows change in steps/day between intervention and control groups from baseline to all reported timepoints for each study. At $\leq 6$ months the pooled estimate of change indicates individuals in the intervention group were doing more steps/day than controls: MD +886 (95\% 


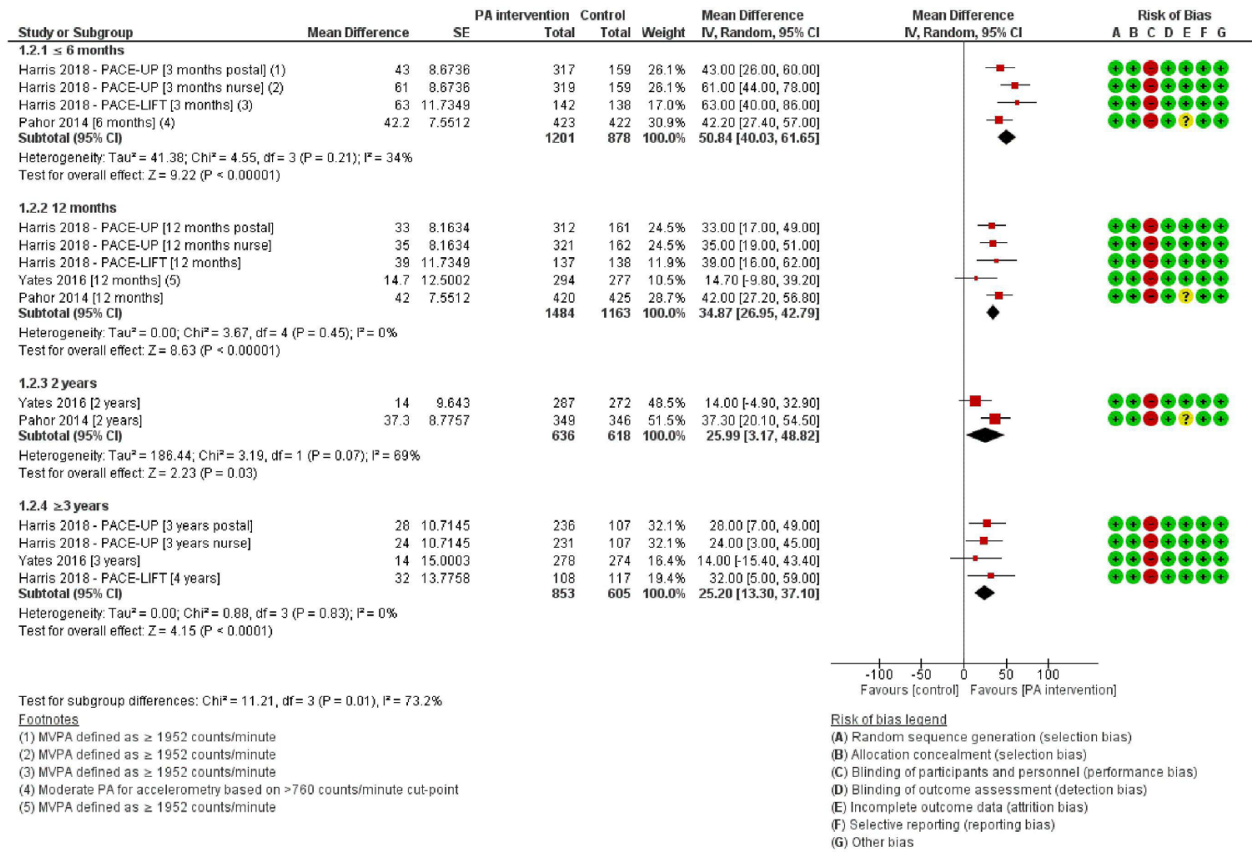

Figure 3 Forest plot showing mean change in Weekly moderate-to-vigorous physical activity.

CI: 610 to $1161 ; \mathrm{I}^{2}=31 \%$; participants $=1878$; three studies). At 12 months individuals in the intervention groups were still doing more steps/day than controls: $\mathrm{MD}+554$ (95\% CI: 384 to $724 ; \mathrm{I}^{2}=0 \%$; participants $=2446$; four studies) . At 2 years only two studies contributed data, the effect estimate was uncertain: $\mathrm{MD}+290$ (95\% CI: -7 to $587 ; \mathrm{I}^{2}=0 \%$; participants=1126; two studies). However, a positive effect was present at $\geq 3$ years with individuals in the intervention groups doing more steps/day than the controls: MD +494 (95\% CI: 251 to $738 ; \mathrm{I}^{2}=0 \%$; participants=1944; four studies). Sensitivity analyses including Varma 2016 made little difference to the estimate (online supplementary additional file 7 ).

\section{Difference in mean change in weekly minutes of MVPA}

A similar pattern is seen for mean change in weekly minutes of MVPA (figure 3). At $\leq 6$ months the pooled estimate of change indicates individuals in the intervention group were doing more minutes of weekly MVPA

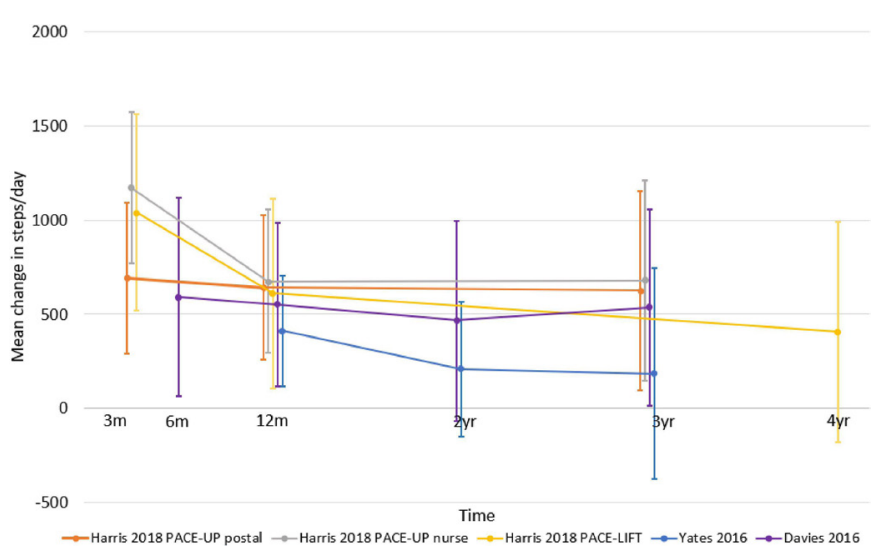

Figure 4 Graph showing individual study data demonstrating mean change in daily steps overtime. than controls: $\mathrm{MD}+51$ (95\% CI: 40 to $62 ; \mathrm{I}^{2}=34 \%$; participants=2079; three studies). At 12 months individuals in the intervention groups were still doing more minutes of weekly MVPA than controls: MD +35 (95\% CI: 27 to 43; $\mathrm{I}^{2}=0 \%$; participants $=2547$; four studies). This effect was sustained at 2 years: $\mathrm{MD}+26$ (95\% CI: 3 to $49 ; \mathrm{I}^{2}=69 \%$; participants=1254; two studies). At 2 years substantial between study heterogeneity was detected, however the test's accuracy is impaired by the small number of studies $(n=2)$ reporting data. There was also a sustained effect at $\geq 3$ years with intervention group participants doing more minutes of weekly MVPA than controls: MD +25 (95\% CI: 13 to $37 ; \mathrm{I}^{2}=0 \%$; participants $=1458$; three studies). Sensitivity analyses including Hays $e t a l^{3}$ attenuated the effect, but left the pattern and conclusions unchanged (online supplementary file 1 ).

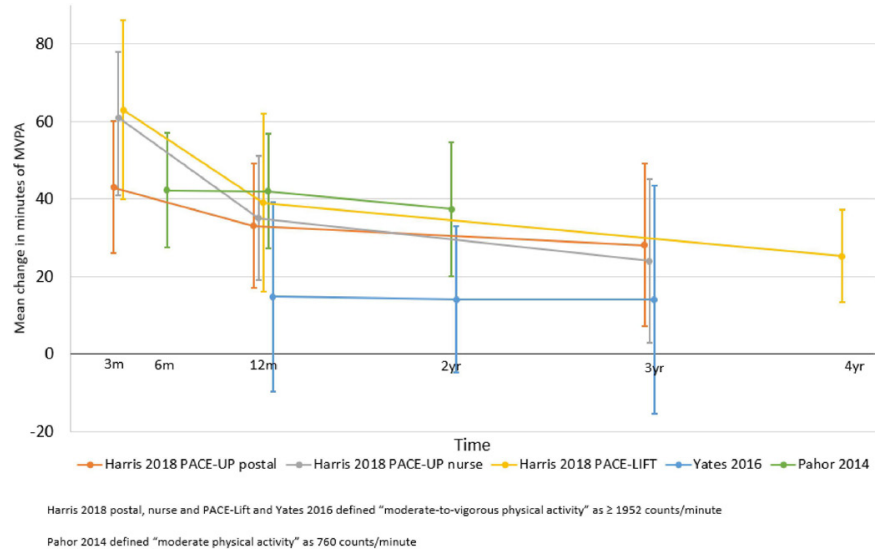

Figure 5 Graph showing individual study data demonstrating mean change in weekly MVPA overtime. MVPA, moderate-to-vigorous physical activity. 
Figures 4 and 5 demonstrate very clearly the consistency of effect over time within each study both for steps (figure 4) and MVPA (figure 5). This consistency is reinforced by the multivariate meta-analysis (online supplementary additional file 8), where MVPA estimates are essentially unchanged, but confidence limits are narrower. For steps/day the estimates were largely similar to the univariate meta-analyses, the notable change being the estimate at 2 years (based on only two studies) which increased from 290 ( -7 to 587) in the univariate analysis, to 479 (244 to 715 ) in the multivariate; again, confidence limits were narrower.

\section{Effects of interventions not included in the main meta-analysis}

Two trials not included in the main meta-analyses, due to data availability, both showed intervention effects beyond 12 months, that is, consistent with meta-analysis findings. Suguira $e t a l^{25}$ found that a 2 year intervention increased mean daily steps in the exercise group compared with the control group (6800-8500 vs 5700-6800 respectively, $\mathrm{p}<0.01)$. Newman et $a l^{24}$ reported that after 18 months the lifestyle intervention group increased their median daily steps compared with controls (8499 vs 6462 respectively, $\mathrm{p}<0.0001)$.

\section{DISCUSSION}

\section{Statement of principal findings}

The review aimed to identify and describe RCTs of PA interventions that measured PA levels objectively and had follow-up beyond 12 months. We adhered to PRISMA guidance throughout the process. Because of the small number of trials identified we were unable to use meta-regression to explore which types of intervention were more successful. Nevertheless, the included studies provided evidence of sustained PA intervention effects beyond 12 months and up to 4 years for both steps (increase of +494 steps/day) and MVPA (increase of +25 min of MVPA weekly) from both individual and groupbased PA interventions. The multivariate meta-analysis emphasised the consistency of step-count and MVPA results.

\section{Strengths and weaknesses of the study}

This review has both strengths and limitations. It was carried out as presented in the prepublished protocol, but due to the small number of studies included, we were unable to perform subgroup and sensitivity analyses to evaluate our secondary aims. Two reviewers independently screened papers at the title, abstract and full-text level and assessed each study for risk of bias. Blinding of participants and personnel is not achievable in interventions of this type, which exposes the trials to a risk of performance bias; although objective outcome measurement mitigates the risk of bias overall. While not all studies clearly described their randomisation or allocation methods, participant attrition or performed an intention to treat analysis, overall the methodological quality of included studies was good. Due to the limited number of studies identified in this review, the generalisability of our evidence is restricted. All included studies were conducted in high-income countries and the majority of participants were of white ethnicity and aged over 40 years old. The interventions varied in their intensity and how pragmatic they would be to implement in realworld settings. Our use of multivariate meta-analysis is an important innovation, allowing us to incorporate data on different outcomes (steps and MVPA) and different time points in a single model improving precision of estimates. With the addition of more studies it could easily be extended to multivariate meta-regression.

\section{Comparison of our findings with other studies}

Several reviews have explored PA maintenance; however, to our knowledge, ours is the first to focus solely on objective PA outcomes beyond 12 months. One systematic review ${ }^{9}$ explored whether behavioural interventions increased PA at 12-36 months in 55-70 year olds; but only 2 of the 21 studies objectively measured PA levels. ${ }^{1011}$ Kuller $e t a l^{10}$ are included in our review as Newman et $a l .{ }^{24}$ We excluded Opdenacker $e t a l^{11}$ due to randomization concerns. Consistent with our findings, this review found that behavioural interventions led to long-term PA improvements at 12 months. ${ }^{9}$ However, they found little evidence for significant intervention effects beyond 12 months and concluded PA maintenance was unclear. ${ }^{9}$ Two recent reviews looked at the effectiveness of PA interventions in achieving behaviour change and maintenance, in healthy inactive adults ${ }^{7}$ and in young and middle-aged adults. ${ }^{33}$ They found clear evidence that PA interventions were effective at maintaining behaviour change after 6 months or more ${ }^{7}$ with interventions having a larger effect on maintenance at 6-9 months compared with 9-15 months. ${ }^{33}$ However, beyond 15 months there was little evidence. The majority of the papers in these two reviews used subjective PA measurements and had follow-up $\leq 12$ months. Our paper builds on and extends findings from all three reviews by identifying more studies with objective PA measures beyond 12 months, and evidence of sustained PA intervention effects up to 4 years.

\section{Implications for clinicians and policy makers}

Our review has important clinical and policy implications. An increase of approximately $35 \mathrm{~min}$ of MVPA weekly at 1 year and approximately $25 \mathrm{~min}$ by $3-4$ years, would contribute substantially towards helping participants meet national PA recommendations of $150 \mathrm{~min}$ of MVPA weekly. Additionally, the greatest health benefits accrue by increasing PA levels in those with very low PA levels, especially if that activity is of moderate or vigorous intensity. ${ }^{34}$ The sustained effect on PA beyond 12 months could therefore produce important long-term health benefits and suggests that investing in community-based PA interventions that achieve long-term effects, would be worthwhile for practitioners and commissioners. Due to insufficient data we cannot be sure exactly what 
interventions are most effective for which participants; however, our findings are based on both individual pedometer-based walking interventions, ${ }^{27}$ individual PA sessions delivered at a centre, supported by home-based activities $^{22}$ and group-based PA interventions. ${ }^{23} 243236$

\section{Future research and unanswered questions}

Our review also has important implications for future research. 'Maintenance of $\mathrm{PA}^{\prime}$ is used and defined in different ways. ${ }^{37}$ A standard definition of PA maintenance would progress understanding of this area. Studies often use different counts/minute cut-points to define MVPA (Harris $e t a l^{27}$ PACE-UP; Harris et $a l^{27}$ PACE-Lift; Pahor et $a l^{22}$; Yates $\left.e t a l^{2}\right)$. Implementing a consistent cut-point would allow for more accurate comparison of results between studies. Given that PA requires regular performance in order to achieve long-term health benefits, future research into PA interventions should be designed with maintenance in mind, for example, by considering potential follow-up time-points, maximising participant engagement to reduce attrition and utilising specific behaviour change techniques promoting long-term habit formation. ${ }^{7}$ The lack of trials with objective PA data beyond 12 months limits our ability to comment on which types of interventions and specifically which intervention components were most likely to achieve long-term behaviour change. More large-scale pragmatic trials in real-world settings with long-term objective PA measures are needed. An increasing number of studies are now objectively measuring PA levels. ${ }^{20}$ It would therefore be beneficial to update this review and meta-analysis when more trials with long-term data are available.

\section{CONCLUSIONS}

There are currently few PA interventions with objective follow-up beyond 12 months. Nevertheless, the studies included in this review provide convincing evidence that, where studies demonstrated short term effects, there were sustained PA intervention effects beyond 12 months and up to 4 years for both steps/day and weekly MVPA. This is an important positive message for tackling the public health inactivity challenge.

Acknowledgements We thank Professor lan White for advice on using Stata procedure mvmeta and to Hays et al and Pahor et al for providing additional data.

Contributors CW, UARC, RF, DGC and TH contributed to the conception of the work and the analysis. All authors contributed to the interpretation of the data. All authors drafted the work revising it critically for important intellectual content and approved the final version to be published. All authors agree to be accountable for all aspects of the work in ensuring that questions related to the accuracy or integrity of any part of the work are appropriately investigated and resolved.

Funding The authors have not declared a specific grant for this research from any funding agency in the public, commercial or not-for-profit sectors.

Competing interests TH and DGC acknowledge conflicts of interest in terms of funding from the National Institute for Health Research for the PACE-Lift and PACEUP trials.

Patient and public involvement Patients and/or the public were not involved in the design, or conduct, or reporting, or dissemination plans of this research.
Patient consent for publication Not required.

Provenance and peer review Not commissioned; externally peer reviewed.

Data availability statement Data are available in a public, open access repository. All data relevant to the study are included in the article or uploaded as supplementary information. Data are shared in our supplementary material and will also be available on the St George's University of London data repository on publication.

Open access This is an open access article distributed in accordance with the Creative Commons Attribution Non Commercial (CC BY-NC 4.0) license, which permits others to distribute, remix, adapt, build upon this work non-commercially, and license their derivative works on different terms, provided the original work is properly cited, appropriate credit is given, any changes made indicated, and the use is non-commercial. See: http://creativecommons.org/licenses/by-nc/4.0/.

ORCID iD

Tess Harris http://orcid.org/0000-0002-8671-1553

\section{REFERENCES}

1 Lee I-M, Shiroma EJ, Lobelo F, et al. Effect of physical inactivity on major non-communicable diseases worldwide: an analysis of burden of disease and life expectancy. Lancet 2012;380:219-29.

2 Warburton DER, Bredin SSD. Health benefits of physical activity: a systematic review of current systematic reviews. Curr Opin Cardiol 2017;32:541-56.

3 Guthold R, Stevens GA, Riley LM, et al. Worldwide trends in insufficient physical activity from 2001 to 2016 : a pooled analysis of 358 population-based surveys with 1.9 million participants. Lancet Glob Health 2018;6:e1077-86.

4 Piercy KL, Troiano RP, Ballard RM, et al. The physical activity guidelines for Americans. JAMA 2018;320:2020-8

5 Health Survey for England. NHS digital, 2016.

6 Bravata DM, Smith-Spangler C, Sundaram V, et al. Using pedometers to increase physical activity and improve health: a systematic review. JAMA 2007;298:2296-304.

7 Howlett N, Trivedi D, Troop NA, et al. Are physical activity interventions for healthy inactive adults effective in promoting behavior change and maintenance, and which behavior change techniques are effective? A systematic review and meta-analysis. Trans/ Behav Med 2019;9:147-57.

8 Reiner M, Niermann C, Jekauc D, et al. Long-term health benefits of physical activity--a systematic review of longitudinal studies. BMC Public Health 2013;13:813.

9 Hobbs N, Godfrey A, Lara J, et al. Are behavioral interventions effective in increasing physical activity at 12 to 36 months in adults aged 55 to 70 years? A systematic review and meta-analysis. BMC Med 2013;11:75.

10 Kuller LH, Kinzel LS, Pettee KK, et al. Lifestyle intervention and coronary heart disease risk factor changes over 18 months in postmenopausal women: the women on the move through activity and nutrition (woman study) clinical trial. $J$ Womens Health 2006;15:962-74.

11 Opdenacker J, Boen F, Coorevits N, et al. Effectiveness of a lifestyle intervention and a structured exercise intervention in older adults. Prev Med 2008;46:518-24.

12 Richards J, Hillsdon M, Thorogood M, et al. Face-To-Face interventions for promoting physical activity. Cochrane Database Syst Rev 2013:Cd010392.

13 Celis-Morales CA, Perez-Bravo F, Ibañez L, et al. Objective vs. self-reported physical activity and sedentary time: effects of measurement method on relationships with risk biomarkers. PLoS One 2012; 7:e36345.

14 Sallis JF, Saelens BE. Assessment of physical activity by self-report: status, limitations, and future directions. Res Q Exerc Sport 2000;71 Suppl 2:1-14.

15 Sylvia LG, Bernstein EE, Hubbard JL, et al. Practical guide to measuring physical activity. J Acad Nutr Diet 2014;114:199-208.

16 Falck RS, McDonald SM, Beets MW, et al. Measurement of physical activity in older adult interventions: a systematic review. $\mathrm{Br} J$ Sports Med 2016;50:464-70.

17 Dyrstad SM, Hansen BH, Holme IM, et al. Comparison of selfreported versus accelerometer-measured physical activity. Med Sci Sports Exerc 2014;46:99-106.

18 Lamb SE, Bartlett HP, Ashley A, et al. Can lay-led walking programmes increase physical activity in middle aged adults? a randomised controlled trial. J Epidemiol Community Health 2002;56:246-52. 
19 Limb ES, Ahmad S, Cook DG, et al. Measuring change in trials of physical activity interventions: a comparison of self-report questionnaire and accelerometry within the PACE-UP trial. Int $J$ Behav Nutr Phys Act 2019;16:10.

20 Silfee VJ, Haughton CF, Jake-Schoffman DE, et al. Objective measurement of physical activity outcomes in lifestyle interventions among adults: a systematic review. Prev Med Rep 2018;11:74-80.

21 Marshall IJ, Noel-Storr A, Kuiper J, et al. Machine learning for identifying randomized controlled trials: an evaluation and practitioner's guide. Res Synth Methods 2018;9:602-614.

22 Pahor M, Guralnik JM, Ambrosius WT, et al. Effect of structured physical activity on prevention of major mobility disability in older adults: the life study randomized clinical trial. JAMA 2014;311:2387-96.

23 Hays LM, Hoen HM, Slaven JE, et al. Effects of a communitybased lifestyle intervention on change in physical activity among Economically disadvantaged adults with prediabetes. Am J Health Educ 2016;47:266-78.

24 Newman MA, Pettee KK, Storti KL, et al. Monthly variation in physical activity levels in postmenopausal women. Med Sci Sports Exerc 2009;41:322-7.

25 Sugiura $\mathrm{H}$, Sugiura $\mathrm{H}$, Kajima K, et al. Effects of long-term moderate exercise and increase in number of daily steps on number of daily steps on serum lipids in women: randomised controlled trial. BMC Women's Health 2002;2:3.

26 Higgins J, Green S. Cochrane Handbook for systematic reviews of interventions version 5.1.0, 2011. Available: www.handbook. cochrane.org

27 Harris T, Kerry SM, Limb ES, et al. Physical activity levels in adults and older adults 3-4 years after pedometer-based walking interventions: long-term follow-up of participants from two randomised controlled trials in UK primary care. PLoS Med 2018;15:e1002526.

28 Singh S. How to conduct and interpret systematic reviews and metaanalyses. Clin Trans/ Gastroenterol 2017;8:e93.

29 White IR. Multivariate Random-effects meta-analysis. Stata J 2009;9:40-56.

30 White IR. Multivariate Random-effects meta-regression: updates to Mvmeta. Stata J 2011;11:255-70.

31 Varma VR, Tan EJ, Gross AL, et al. Effect of community volunteering on physical activity: a randomized controlled trial. Am J Prev Med 2016;50:106-10.

32 Yates T, Edwardson CL, Henson J, et al. Walking away from type 2 diabetes: a cluster randomized controlled trial. Diabet Med 2017;34:698-707.

33 Murray JM, Brennan SF, French DP, et al. Effectiveness of physical activity interventions in achieving behaviour change maintenance in young and middle aged adults: a systematic review and metaanalysis. Soc Sci Med 2017;192:125-33.

34 Thompson PD, Eijsvogels TMH. New physical activity guidelines: a call to activity for clinicians and patients. JAMA 2018;320:1983-4.

35 Wahid A, Manek N, Nichols M, et al. Quantifying the association between physical activity and cardiovascular disease and diabetes: a systematic review and meta-analysis. J Am Heart Assoc 2016;5:e002495.

36 Davies MJ, Gray LJ, Troughton J, et al. A community based primary prevention programme for type 2 diabetes integrating identification and lifestyle intervention for prevention: the let's prevent diabetes cluster randomised controlled trial. Prev Med 2016;84:48-56.

37 Kahlert D. Maintenance of physical activity: do we know what we are talking about? Prev Med Rep 2015;2:178-80. 